Pacific Journal of Mathematic 


\title{
ON A CLASS OF POLYNOMIALS ORTHOGONAL OVER A DENUMERABLE SET
}

\author{
D. J. Dickinson, H. O. Pollak and G. H. Wannier
}

1. Introduction and Summary. This study proceeds from a theorem of Favard that states that polynomial sets obeying a certain type of three-term recursion formula are, with respect to some weight function, orthogonal over some set on the real axis. For a rather wide subclass of these polynomial sets, it is shown that the orthogonality set consists of a discrete but infinite set of points of the real axis. The orthogonality set and the weight function are given by certain entire functions that can be constructed from the polynomials by a limiting process. A set of modified Lommel polynomials is given as an explicit example of the general theory. They are orthogonal over the reciprocals of the roots of certain Bessel functions.

2. General theory and existence of orthogonality. In 1935, Favard [2] sketched the proof of the following theorem:

If there is a sequence of real polynomials $\left\{\varphi_{n}(x)\right\}$ possessing a recursion relation of the form

$$
\varphi_{n+1}(x)=\left(x-a_{n}\right) \varphi_{n}(x)-\lambda_{n} \varphi_{n-1}(x), \quad(n \geqq 0),
$$

with

$$
\varphi_{0}(x)=1, \quad \varphi_{1}(x)=x-a_{0}, \quad \varphi_{-1}(x)=0
$$

and

$$
\lambda_{n}>0
$$

then the polynomials are orthogonal.

This powerful theorem seems to have escaped attention; it suggests that whenever a relation of the form (1) exists, a weight function and orthogonality domain could probably be found by detailed study. We want to accomplish this for a wide class of polynomials for which the orthogonality range comes out to be a denumerable set of points.

The class of polynomials mentioned is obtained by imposing upon (2) and (3) the restrictions that

$$
a_{n}=0
$$

and that the limit $B$ exists where

Received December 28, 1953, and in revised form April 15, 1955. 


$$
\sum_{n=1}^{\infty} \lambda_{n}=B
$$

The restriction (4) implies that the polynomials are alternately even and odd and that we may take our weight function $d \mu(x)$ such that

$$
\int_{-\infty}^{+\infty} x^{2 n+1} d \mu(x)=0
$$

for positive integral $n$. We may take infinite limits of integration since, by defining some factor of the integrand as zero at suitable points, all real integrals may be written with infinite limits. We also take $\mu(x)$ to be a nondecreasing function such that

$$
\int_{-\infty}^{+\infty} d \mu(x)=1
$$

From (1) and (2), the leading coefficients of our polynomials are unity and thus

$$
\int_{-\infty}^{+\infty} x \varphi_{n-1}(x) \varphi_{n}(x) d \mu(x)=\int_{-\infty}^{+\infty} \varphi_{n}^{2}(x) d \mu(x) .
$$

Hence after multiplying (1) by $\varphi_{n-1}(x) d \mu(x)$ and integrating we have

$$
0=\int_{-\infty}^{\infty} \varphi_{n}^{2}(x) d \mu(x)-\lambda_{n} \int_{-\infty}^{\infty} \varphi_{n-1}^{2}(x) d \mu(x), \quad(n>0) .
$$

The solution of this recursion relation subject to the condition (6) is

$$
\int_{-\infty}^{+\infty} \varphi_{n}^{2}(x) d \mu(x)=\prod_{i=1}^{n} \lambda_{i}
$$

where for $n=0$, the product on the right is to be taken as unity.

We now introduce a second set of polynomials $F_{n}(z)$ by the substitutions

$$
\begin{gathered}
z=1 / x, \\
F_{n}(z)=z^{n} \varphi_{n}(1 / z) .
\end{gathered}
$$

Because of (1), these polynomials obey the recursion relation

$$
F_{n+1}(z)-F_{n}(z)=-\lambda_{n} z^{2} F_{n-1}(z),
$$

We deduce from this relation the following

THEOREM 1. There exists an entire function $F(z)$ such that $\left|F_{n}(z)\right| \leqq F(z)$ for all $n$ and real $z$.

Proof. We define $G_{n}(z)$ as the larger of the absolute values of $F_{n}(z)$ 
and $F_{n-1}(z)$. This, with (10), yields

$$
\left|F_{n+1}(z)\right| \leqq\left(1+\lambda_{n}|z|^{2}\right) G_{n}(z),
$$

and hence

$$
G_{n+1}(z) \leqq\left(1+\lambda_{n}|z|^{2}\right) G_{n}(z)
$$

From this and the easily verified identities

$$
F_{0}(z)=F_{1}(z)=G_{1}(z)=1,
$$

we have, for all $n$,

$$
\left|F_{n}(z)\right| \leqq \prod_{n=1}^{\infty}\left(1+\lambda_{n}|z|^{2}\right)
$$

Here the convergence of the infinite product is assured by (5). If we define an entire function $F(z)$ by

$$
F(z)=\prod_{n=1}^{\infty}\left(1+\lambda_{n} z^{2}\right)
$$

then we have, for real $z$ and all $n$, that

$$
\left|F_{n}(z)\right| \leqq F(z) \text {. }
$$

THEOREM 2. The polynomials $F_{n}(z)$ converge toward an entire function $E(z)$ whose zeros form a denumerable set on the real axis. For all $z$ in any bounded set, the convergence is uniform.

Proof. We first derive the Cauchy criterion for the set using equations (10) and (5) and Theorem 1.

$$
\begin{aligned}
\left|F_{n+M}(z)-F_{n}(z)\right| & \leqq \sum_{i=0}^{M-1}\left|F_{n+M-i}(z)-F_{n+y-1-i}(z)\right| \\
& \leqq|z|^{2} F(z) \sum_{i=0}^{M-1} \lambda_{n+H-1-i} \\
& \leqq|z|^{2} F(z)[S(n)-S(n+M)]
\end{aligned}
$$

where

$$
S(n)=\sum_{i=n}^{\infty} \lambda_{i} .
$$

Since $S(n)$ is a convergent series, the analytic functions $F_{n}(z)$ converge uniformly toward a function $E(z)$ if $z$ is bounded. Hence $E(z)$ is an analytic entire function of $z$. It cannot vanish identically because from (2), (9), and (10), we have that

$$
E(0)=1 \text {. }
$$


From a theorem of Hurwitz (see [4], p. 119 and [3], Theorem 1.91.3) it follows that if $z_{\jmath}$ is a zero of $E(z)$ of multiplicity $q$ and $N\left(z_{0}\right)$ some neighborhood of $z_{0}$, then for $n$ sufficiently large, $N\left(z_{0}\right)$ contains precisely $q$ zeros of $F_{n}(z)$. Now for any positive integer $p$, there exists a circle $C_{p}$ centered on the origin that has in its exterior at least $p$ zeros of $\varphi_{p+1}(x)$. From the Sturmian properties of the $\varphi_{n}(x)$, it follows that for $n>p+1, \varphi_{n}(x)$ also has at least $p$ zeros in the exterior of $C_{p}$. That is, there exists a circle $C_{p}^{\prime}$ (the reciprocal circle of $C_{p}$ ) that contains in its interior at least $p$ zeros of each of the polynomials $F_{n}(z)$ for $n>p+1$. Hence for this arbitrary integer $p, E(z)$ contains at least $p$ zeros in or on $C_{p}^{\prime}$. (A zero of multiplicity $q$ we count as $q$ zeros.) Thus $E(z)$ has an infinite number of zeros.

The reality of the zeros of the $F_{n}(z)$ can be seen from the Sturmian properties of the $\varphi_{n}(x)$ and, using the Hurwitz theorem again, it quickly follows that the zeros of $E(z)$ are real.

THEOREm 3. The derivative of $\mu(x)$ is everywhere zero except at the reciprocals of the zeros of $E(z)$.

A theorem of this type appears in the literature. (See [3], Theorem 6.1.1.) In the following a proof applicable to the present situation is given.

Proof. From Theorem 2 and (9), it follows that

$$
\lim _{n \rightarrow \infty} \frac{\varphi_{n}(x)}{x^{n}}=E\left(\frac{1}{x}\right)
$$

uniformly in every bounded domain.

Suppose we fix our attention on the interval between two successive positive zeros, $p_{\nu}$ and $p_{\nu+1}$ of $E(1 / x)$. Let us consider an interval slightly smaller than this, $[a, b]$, so that $E^{2}(1 / x)$ will have a positive lower bound over the interval. We may then use the two preceding theorems to develop the following estimate:

$$
\begin{aligned}
\int_{a}^{b} x^{2 n} & E^{2}(1 / x) d \mu(x) \\
\quad & =\int_{a}^{b} \varphi_{n}^{2}(x) d \mu(x)+\int_{a}^{b}\left[x^{2 n} E^{2}(1 / x)-\varphi_{n}^{2}(x)\right] d \mu(x) \\
& \leqq \int_{a}^{b} \varphi_{n}^{2}(x) d \mu(x)+\int_{a}^{b} x^{2 n}\left|E(1 / x)-F_{n}(1 / x)\right| \cdot|E(1 / x)+F(1 / x)| d \mu(x) \\
& \leqq \int_{a}^{b} \varphi_{n}^{2}(x) d \mu(x)+2 S(n) \int_{a}^{b} x^{2(n-1)} F^{n}(1 / x) d \mu(x) .
\end{aligned}
$$

This may be written in the form 


$$
\int_{a}^{b} x^{2 n}\left\{E^{2}(1 / x)-\frac{2 S(n)}{x^{2}} F^{2}(1 / x)\right\} d \mu(x) \leq \int_{a}^{b} \varphi_{n}^{2}(x) d \mu(x) .
$$

Now we can choose $n$ sufficiently large to make the term in the bracket on the left positive; for the interval was chosen so as to give $E^{\prime 2}(1 / x)$ a lower bound and the negative term contains the convergent factor $S(n)$. The inequality is then preserved if we replace the factor $x^{2 n}$ by $a^{2 n}$. On the right-hand side, we may replace the limits by $-\infty$ and $+\infty$ and use (5) and (7) to estimate the normalization integral. This yields

$$
\int_{a}^{b} E^{2}(1 / x) d \mu(x) \leqq 2 S(n) \int_{a}^{b} \frac{1}{x^{2}} F^{2}(1 / x) d \mu(x)+\frac{1}{a^{2 n}} \prod_{i=1}^{n} \lambda_{i} .
$$

Both the right-hand terms tend to zero as $n$ increases while the left-hand side is independent of $n$. Hence, the integral must vanish identically. This reasoning can be repeated for every interval between the roots of $E(1 / x)$. Hence, the contribution to $\frac{d \mu}{d x}$ arises entirely from the zeros as stated by the theorem.

THEOREM 4. The polynomials $\varphi_{n}(x)$ are orthogonal over a denumerable set of points of the real axis.

This is a trivial consequence of Theorem 3 and the properties of $E(z)$ enumerated in Theorem 2.

3. Construction of the weight function. We first need to generalize some of the previous definitions.

We will consider the polynomials that satisfy

$$
\varphi_{n+1}^{(s)}(x)=x \varphi_{n}^{(s)}(x)-\lambda_{n+s} \varphi_{n-1}^{(s)}(x),
$$

where $s$ is a nonnegative integer, where $\lambda_{n}$ is restricted as in (3) and (5), and where

$$
\varphi_{1}^{(s)}(x)=x, \varphi_{0}^{(s)}(x)=1, \varphi_{-1}^{(s)}(x)=0 .
$$

From (9) and (10) we have

$$
F_{n+1}^{(s)}(z)=F_{n}^{(s)}(z)-\lambda_{n+s} z^{2} F_{n-1}^{(s)}(z), \quad(n \geq 0),
$$

where

$$
F_{n}^{(s)}(z)=z^{n} \varphi_{n}^{(s)}(1 / z) .
$$

Let us obtain a contiguous function recurrence relation for the $F_{n}^{(s)}(z)$. From (16), we may write 


$$
\begin{array}{ll}
F_{n+1}^{(s)}(z)=F_{n}^{(s)}(z)-\lambda_{n+s} z^{2} F_{n-1}^{(s)}(z), & (n \geqq 0), \\
F_{n}^{(s+1)}(z)=F_{n-1}^{(s+1)}(z)-\lambda_{n+s} z^{2} F_{n-2}^{(s+1)}(z), & (n \geqq 1), \\
F_{n-1}^{(s+2)}(z)=F_{n-2}^{(s+2)}(z)-\lambda_{n+s} z^{2} F_{n-3}^{(s+2)}(z), & (n \geq 2) .
\end{array}
$$

Now if we define $\psi_{n}^{(s)}(z)$ by

$$
\psi_{n+1}^{(s)}(z)=A(z, s) F_{n+1}^{(s)}(z)+B(z, s) F_{n}^{(s+1)}(z)+C(z, s) F_{n-1}^{(s+2)}(z), \quad(n \geqq 0),
$$

where $A(z, s), B(z, s)$, and $C(z, s)$ are arbitrary functions independent of $n$, we have that

$$
\psi_{n+1}^{(s)}(z)=\psi_{n}^{(s)}(z)-\lambda_{n+s} z^{2} \psi_{n-1}^{(s)}(z), \quad(n \geq 2) .
$$

Let us set

$$
\begin{aligned}
& A(z, s)=1, \\
& B(z, s)=-1, \\
& C(z, s)=\lambda_{s+1} z^{2} .
\end{aligned}
$$

It follows, using (15) and (17), that $\psi_{1}^{(s)}(z)=0$ and $\psi_{2}^{(s)}(z)=0$. Thus we must have that $\psi_{n}^{(s)}(z)=0$ for $n \geqq 1$. That is, we have the contiguous function recurrence relation

$$
F_{n}^{(s)}(z)-F_{n-1}^{(s+1)}(z)+\lambda_{s+1} z^{2} F_{n-2}^{(s+2)}(z)=0, \quad(n \geqq 1) .
$$

Now we have that

$$
\lim _{n \rightarrow \infty} F_{n}^{(s)}(z)=E^{(s)}(z),
$$

where $E^{(s)}(z)$ is an entire function of the sort described in Theorem 2. The recurrence relation for these entire functions

$$
E^{(s)}(z)-E^{(s+1)}(z)+\lambda_{s+1} z^{2} E^{(s+2)}(z)=0
$$

follows from (18) and (19).

Now (18) and (20) look very similar. We may exploit this similarity to obtain a relation between the polynomials and the entire functions. If we multiply (18) by $E^{(s+1)}(z)$, multiply (20) by $F_{n-1}^{(s+1)}(z)$, and subtract the resulting expressions, we obtain an expression that may be written

$$
\begin{aligned}
& {\left[E^{(s+1)}(z) F_{n}^{(s)}(z)-E^{(s)}(z) F_{n-1}^{(s+1)}(z)\right]} \\
& \quad=\left[E^{(s+2)}(z) F_{n-1}^{(s+1)}(z)-E^{(s+1)}(z) F_{n-2}^{(s+2)}(z)\right] \lambda_{s+1} z^{2} .
\end{aligned}
$$

Here, the similarity of the brackecied terms suggests iteration. Iterating it $(n-1)$ times, we obtain 


$$
\begin{aligned}
& {\left[E^{(s+1)}(z) F_{n}^{(s)}(z)-E^{(s)}(z) F_{n-1}^{(s+1)}(z)\right]} \\
& \quad=\left[E^{(s+1+n)}(z) F_{0}^{(s+n)}-E^{(s+n)}(z) F_{-1}^{(s+1+n)}(z)\right] z^{2 n} \prod_{i=1}^{n} \lambda_{s+i} \\
& \quad=E^{(s+1+n)}(z) z^{2 n} \prod_{i=1}^{n} \lambda_{s+i} .
\end{aligned}
$$

From this we may form the expression

$$
\begin{aligned}
z^{-n-p-1} F_{n}^{(s)}(z) & \left(\frac{E^{(s+1)}(z)}{E^{(s)}(z)}\right) \\
= & z^{-n-p-1} F_{n-1}^{(s+1)}(z)+\left(\frac{E^{(s+n+1)}(z)}{E^{(s)}(z)}\right) z^{n-p-1} \prod_{i=1}^{n} \lambda_{s+i} .
\end{aligned}
$$

Let us consider the residues at the origin of the right member of (21). The residue of the first term of this right member is zero for $p \geqq 0$, ( $p$ integral). The quotient of the entire functions that appears in the second term is unity at the origin. Hence, the residue of this second term is zero for $n-p>0$ and is not zero for $p=n$. Thus we have

$$
2 \pi i \int_{c^{\prime}} z^{-n-p-1} F_{n}^{(s)}(z)\left(\frac{E^{(s+1)}(z)}{E^{(s)}(z)}\right) d z= \begin{cases}\prod_{i=1}^{n} \lambda_{s+i} & \text { for } p=n, \\ 0 & \text { for } p<n,\end{cases}
$$

where again the product on the right is taken to be unity when $n=0$, and where the integration is taken counterclockwise around a circle $c^{\prime}$ that includes the origin but does not include any other singularities of the integrand.

If now in (22) we set $x \rightarrow z^{-1}$ and use (17), we have

$$
\frac{1}{2 \pi i} \int_{c} x^{p} \varphi_{n}^{(s)}(x)\left(\frac{E^{(s+1)}(1 / x)}{x E^{(s)}(1 / x)}\right) d x= \begin{cases}\prod_{i=1}^{n} \lambda_{s+i} & \text { for } p=n, \\ 0 & \text { for } p<n,\end{cases}
$$

where the integration now is taken counterclockwise around a curve $c$ that includes all of the singularities of the integrand.

It follows from the Sturmian properties of the polynomials of (18) and from the limit (19) that the weight function in the brackets of (23) has positive residues at all its poles. When (23) is now expressed as the sum of the residues of the integrand, we have

$$
\int_{-a(s)}^{+a(s)} x^{p} \varphi_{n}^{(s)}(x) d \alpha^{(s)}(x)= \begin{cases}\prod_{i=1}^{n} \lambda_{i+s} & \text { for } n=p, \\ 0 & \text { for } p<n,\end{cases}
$$

where $\alpha^{(s)}(x)$ is a monotonic, nondecreasing function whose increase at the point $x$ is equal to the residue of 


$$
\frac{E^{(s+1)}(1 / x)}{x E^{(s)}(1 / x)}
$$

at the point $x$. The limits of integration are the bounds of an interval large enough to contain the zeros of $E^{(s)}(1 / x)$. Since (12) is bounded away from zero, these integration limits may be taken as finite. Again, when $n=0$, we define the product on the right to be unity.

We have thus proved the following

THEOREM 5. Let

where

$$
\varphi_{n+1}^{(s)}(x)=x \varphi_{n}^{(s)}(x)-\lambda_{n+s} \varphi_{n-1}^{(s)}(x), \quad(n \geq 0), s \geq 0,
$$

$$
\lambda_{n}>0, \sum_{n=1}^{\infty} \lambda_{n}=B<\infty \quad \text { and }
$$

$$
\varphi_{1}^{(s)}(x)=x, \varphi_{0}^{(s)}(x)=1, \varphi_{-1}^{(s)}(x)=0 .
$$

Let, in addition,

$$
\lim _{n \rightarrow \infty} \frac{\varphi_{n}^{(s)}(x)}{x^{n}}=E^{(s)}\left(\begin{array}{l}
1 \\
x
\end{array}\right)
$$

Then

$$
\int_{-a(s)}^{+a(s)} x^{y} \varphi_{n}^{(s)}(x) d \alpha^{(s)}(x)= \begin{cases}\prod_{i=1}^{n} \lambda_{i+s} & \text { for } n=p, \\ 0 & \text { for } p<n,\end{cases}
$$

where $\alpha^{(s)}(x)$ is a monotonic non-decreasing function whose increase at the point $x$ is equal to the residue of

$$
\frac{E^{(s+1)}(1 / x)}{x E^{(s)}(1 / x)}
$$

at the point $x$. The limits of integration are the bounds of an interval large enough to contain the zeros of $E^{(s)}(1 / x)$.

COROLLARY. Under the same conditions as Theorem 5,

$$
\int_{-a(s)}^{a(s)} \varphi_{m}^{(s)}(x) \varphi_{n}^{(s)}(x) d \alpha^{(s)}(x)= \begin{cases}\prod_{i=1}^{n} \lambda_{i+s} & \text { for } n=m, \\ 0 & \text { for } n \neq m .\end{cases}
$$

4. An example. An illustration of our theory is given by a set of modified Lommel polynomials. They may be defined by the recurrence relation 


$$
L_{n-1}^{(s)}(x)=x L_{n}^{(s)}(x)-\frac{1}{4(s+n)(s+n+1)} L_{n-1}^{(s)}(x) \quad n \geq 0, s>0
$$

together with

$$
L_{0}^{(s)}=1, L_{-1}^{(s)}(x)=0
$$

In terms of Watson's [5] definition of the Lommel polynomial $R_{n, \nu}(x)$, we have

$$
L_{n}^{(s)}(x)=\frac{R_{n, s+1}(1 / x) s !}{2^{n}(s+n) !}
$$

The special case of the limit (19),

$$
\lim _{n \rightarrow \infty} x^{n} L_{n}^{(s)}(1 / x)=\frac{(x / 2)^{s}}{s !} J_{s}(x)
$$

may be found in Watson [5], §9.65.

The residue of

$$
\frac{J_{s+1}(1 / x)}{2 x^{2}(s+1) J_{s}(1 / x)}
$$

at $x_{z}$, a zero of $J_{s}(1 / x)$ is $2(s+1) x_{z}^{2}$.

Thus we have that

$$
2(s+1) \sum x^{p} L_{n}^{(s)}(x) x^{2}= \begin{cases}\frac{s !(s+1) !}{4^{n}(s+n) !(s+n+1) !} & \text { for } p=n, \\ 0 & \text { for } p<n,\end{cases}
$$

where the summation is taken over all $x$ that are finite zeros of $J_{s}(1 / x)$.

The result (25) has also been obtained using a somewhat different approach [1].

\section{REFERENCES}

1. D. J. Dickinson, On Bessel and Lommel polynomials, to appear in Proc. Amer. Math. Soc.

2. J. Favard, Sur les polynomes de Tchebicheff, C. R. Acad. Sci. Paris, 200 (1935), 2052-2053.

3. G. Szegö, Orthogonal polynomials, Amer. Math. Soc. Colloquium Publication, vol. 23, New York, 1939.

4. E. C. Titchmarsh, The theory of functions, second edition, Oxford University Press, London, 1939.

5. G. N. Watson, A treatise on the theory of Bessel functions, second edition, Cambridge, 1952. 



\section{PACIFIC JOURNAL OF MATHEMATICS}

EDITORS

\author{
H. L. Royden \\ Stanford University \\ Stanford, California \\ E. Hewite \\ University of Washington \\ Seattle 5 , Washington
}

\author{
R. P. Dilworth \\ California Institute of Technology \\ Pasadena 4, California
}

\author{
A. Horn* \\ University of California \\ Los Angeles 24, California
}

\section{ASSOCIATE EDITORS}
E. F. BECKENBACH
M. HALL
M. S. KNEBELMAN
J. J. STOKER
C. E. BURGESS
P. R. HALMOS
I. NIVEN
V. GANAPATHY IYER
T. G. OSTROM
G. SZEKERES
H. BUSEMANN
R. D. JAMES
M. M. SCHIFFER
F. WOLF
H. FEDERER

\section{SPONSORS}

\author{
UNIVERSITY OF BRITISH COLUMBIA \\ CALIFORNIA INSTITUTE OF TECHNOLOGY \\ UNIVERSITY OF CALIFORNIA \\ CALIFORNIA RESEARCH CORPORATION \\ MONTANA STATE UNIVERSITY \\ UNIVERSITY OF NEVADA \\ OREGON STATE COLLEGE \\ UNIVERSITY OF OREGON \\ UNIVERSITY OF SOUTHERN CALIFORNIA
}

\author{
STANFORD UNIVERSITY \\ UNIVERSITY OF UTAH \\ WASHINGTON STATE COLLEGE \\ UNIVERSITY OF WASHINGTON \\ AMERICAN MATHEMATICAL SOCIETY \\ HUGHES AIRCRAFT COMPANY
}

Mathematical papers intended for publication in the Pacific Journal of Mathematics should be typewritten (double spaced), and the author should keep a complete copy. Manuscripts may be sent to any of the editors. Manuscripts intended for the outgoing editors should be sent to their successors. All other communications to the editors should be addressed to the managing editor, Alfred Horn at the University of California, Los Angeles 24, California.

50 reprints of each article are furnished free of charge; additional copies may be obtained at cost in multiples of 50 .

The Pacific Journal of Mathematics is published quarterly, in March, June, September, and December. The price per volume (4 numbers) is $\$ 12.00$; single issues, $\$ 3.50$. Back numbers are available. Special price to individual faculty members of supporting institutions and to individual members of the American Mathematical Society: $\$ 4.00$ per volume; single issues, $\$ 1.25$.

Subscriptions, orders for back numbers, and changes of address should be sent to Pacific Journal of Mathematics, c/o University of California Press, Berkeley 4, California.

Printed at Kokusai Bunken Insatsusha (International Academic Printing Co., Ltd.), No. 10, 1-chome, Fujimi-cho, Chiyoda-ku, Tokyo, Japan.

* During the absence of E. G. Straus.

PUBLISHED BY PACIFIC JOURNAL OF MATHEMATICS, A NON-PROFIT CORPORATION COPYRIGHT 1956 BY PACIFIC JOURNAL OF MATHEMATICS 


\section{Pacific Journal of Mathematics}

\section{Vol. 6, No. $2 \quad$ December, 1956}

Louis Auslander, Remark on the use of forms in variational calculations .......................................... 209

Hubert Spence Butts, Jr. and Henry B. Mann, Corresponding residue systems in algebraic number fields ........................ 211

L. Carlitz and John Herbert Hodges, Distribution of matrices in a finite field............................................

Paul Civin and Bertram Yood, Invariant functionals ............... 231

David James Dickinson, Henry Pollak and G. H. Wannier, On a class of polynomials orthogonal over a denumerable set .................

Bernard Friedman and Luna Mishoe, Eigenfunction expansions associated with a non-self-adjoint differential equation ....................

Luna Mishoe and G. C. Ford, On the uniform convergence of a certain eigenfunction series .............................. 271

John W. Green, Mean values of harmonic functions on homothetic curves...........................................

Charles John August Halberg, Jr. and Angus E. Taylor, On the spectra of linked operators .....................................

Chuan Chih Hsiung, Some integral formulas for closed hypersurfaces in Riemannian space ................................... 291

Norman D. Lane, Differentiable points of arcs in conformal n-space 301

Louis F. McAuley, A relation between perfect separability, completeness, and normality in semi-metric spaces

G. Power and D. L. Scott-Hutton, The slow shearing motion of a liquid past a semi-infinite plane .............................

A. C. Schaeffer, Entire functions

Edward Silverman, An intrinsic inequality for Lebesgue area...

Choy-Tak Taam, Asymptotic relations between systems of differential equations.

Ti Yen, Quotient algebra of a finite $A W^{*}$-algebra ... 